\title{
Recursive Singular Spectrum Analysis for Induction Machines Unbalanced Rotor Fault Diagnosis
}

\author{
Vahid Abolghasemi, Senior Member, IEEE, Mohammad Hoseintabar Marzebali, Member, IEEE, \\ and Saideh Ferdowsi, Member, IEEE
}

\begin{abstract}
One of the major challenges of diagnosing rotor symmetry faults in induction machines is severe modulation of fault and supply frequency components. In particular, existing techniques are not able to identify fault components in the case of low slips. In this paper, this problem is tackled by proposing a novel approach. First, a new use of singular spectrum analysis (SSA), as a powerful spectrum analyser, is introduced for fault detection. Our idea is to treat the stator current signature of the wound rotor induction machine as a time series. In this approach, the current signature is decomposed into several eigenvalue spectra (rather than frequency spectra) to find a subspace where the fault component is recognisable. Subsequently, the fault component is detected using some data-driven filters constructed with the knowledge about characteristics of supply and fault components. Then, an inexpensive peak localisation procedure is applied to the power spectrum of the fault component to identify the exact frequency of the fault. The fault detection and localisation methods are then combined in a recursive regime to further improve the diagnosis' performance particularly at high rotor speeds and small rotor faults. The proposed approach is data-driven and is directly applied to the raw signal with no suppression or filtration of the frequency harmonics with a low computational complexity. The numerical results obtained with real data at several rotation speeds and fault severities, unveil the effectiveness and real-time feature of the proposed approach.
\end{abstract}

Index Terms-Singular spectrum analysis, power spectrum, induction machine, unbalanced rotor fault.

\section{INTRODUCTION}

D IAGNOSING mechanical and electrical faults in induction machines (IMs) are necessary o reduce maintenance cost and downtime. This is of particular importance due to the extensive utilisation of these machines in various applications such as automotive, mining, manufacturing, railway, and agriculture, where machine malfunction is a major failure causing huge economical losses [1]. Different types of faults can occur in induction machines as a result of the harsh working conditions. These are mainly categorised as bearing fault, unbalanced rotor winding fault, eccentricity fault, and broken rotor bar [2]. Many condition monitoring techniques have been reported in the literature including invasive and noninvasive approaches. Today, non-invasive approach is widely preferred as it does not require any external sensors on IMs, thus, considered as a cost-effective technique [3]. Among these, motor-current signature analysis (MCSA) is considered the most popular and effective one. This is mainly because the current sensors are relatively inexpensive and one can

V. Abolghasemi (v.abolghasemi@essex.ac.uk) and S. Ferdowsi (s.ferdowsi@essex.ac.uk) are with the School of Computer Science and Electronic Engineering, University of Essex, UK.

M. Hoseintabar Marzebali (m.hoseintabar@shahroodut.ac.ir) is with the Faculty of Electrical Engineering and Robotics, Shahrood University of Technology, Shahrood, Iran. easily access the stator and rotor currents to extract the fault frequencies.

One of the electrical faults that affect the performance and dynamic behaviour of a machine is related to the inter-turn and unbalance fault in the rotor and stator windings. This type of fault in rotor winding of wound-rotor induction machines (WRIM) induces fault harmonic components in the stator current which are modulated as sidebands around the supply frequency. These additive frequencies in the stator current spectrum occur at [4], [5]:

$$
f_{i}=(1 \pm 2 k s) f_{s}, \quad k=1,2,3, \ldots
$$

where $f_{s}$ and $s$ are supply frequency and slip, respectively. Based on (1), the range of fault variations with considering no-load slip $\left(s_{n l} \approx 0\right)$ and rated slip of WRIM $\left(s_{\text {rated }}\right)$ is $\left[\begin{array}{lll}\left(1-2 s_{\text {rated }}\right) f_{s} & f_{s}\end{array}\right]$. It means that in a lower operational slip of motor in the presence of the unbalanced fault in the rotor winding of WRIM, the fault component frequency will be closer to the main frequency (supply frequency). Therefore, diagnosing fault location and its discrimination can be more difficult in the case of low slips. A simple way to emulate the unbalanced rotor fault is to insert an additional resistance in series, denoted by $R_{u n b}$, to one phase stator/rotor winding [6].

Fourier analysis (FA) is a common classic approach in this context. However, this technique has some limitations such as frequency resolution, noise, spectrum leakage and loss of time evaluation, and it fails as slip varies, particularly when the unbalanced fault $R_{u n b}$ is small [6]. For clarity, power spectrum of two 12.8 seconds' healthy and faulty current signals (equivalent to 40,000 points at sampling frequency $F_{s m}=2500$ $\mathrm{Hz})$ with $R_{\text {unb }}=0.003$ p.u. and $s=0.06(1450 \mathrm{rpm})$ are shown in Figure 1. The power spectrum was preceded with a Hanning window to reduce the effects of spectral leakage. As found from Figure 1, the fault component at around 46.66 $\mathrm{Hz}$ is not visible from the spectrum which challenges the FA. Another drawback is that FA is only applicable when enough samples (points) are captured before calculating the power spectrum. This limits the ability to perform real-time condition monitoring which is crucial to the immediate fault detection process and unwanted downtime in industrial machines [7]. Figure 2 compares the power spectrum of one faulty signal, preceded with a Hanning window, under different acquisition intervals (6 and 12.8 seconds) with the same sampling rates. It is found from this figure that although fault is recognisable by inspection of a 40000-point FFT (fast Fourier transform), it is weakened significantly when the frequency resolution is decreased (Figure 2 (b)).

Existing related studies showed that stator and rotor currents are directly affected by asymmetry in the rotor and stator winding of WRIM, respectively [8]-[11]. Hedayati Kia, 


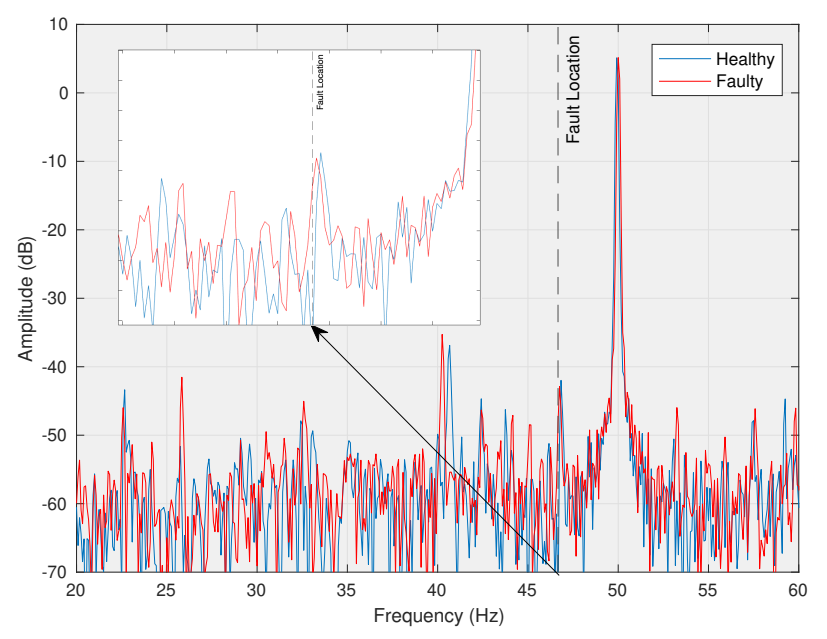

Fig. 1. Healthy (blue) and faulty (red) power spectrum of sample current signals at $s=0.03(1450 \mathrm{rpm})$ and $R_{u n b}=0.003$ p.u. The fault has occurred at $46.66 \mathrm{~Hz}$ (dashed line), however, it is not recognisable using Fourier analysis as can be noticed in the magnified region.

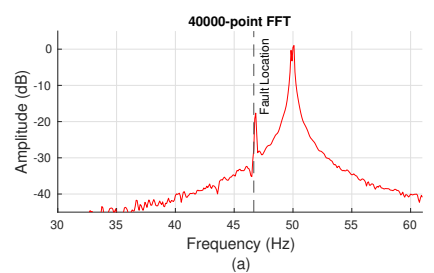

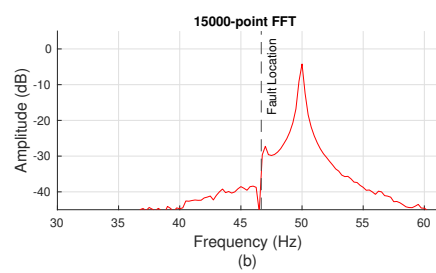

Fig. 2. Comparison between two different faulty spectra with $s=0.03$ (1450 rpm) and $R_{u n b}=0.156$ p.u. (a) and (b) show 40000 -point (12.8 seconds) and 15000 -point (6 seconds) FFT of current the same signal, respectively. While the fault location is visible in (a), it can be barely recognised be inspecting (b).

[8], showed that the energy of a smoothed current in a healthy WRIM would be significantly smaller than that for a faulty one. Hence, discrete wavelet transform (DWT) as timefrequency/time-scale signal processing was used to estimate and remove the DC current components. Since fault frequency is so close to the main frequency under the rotor asymmetry fault, and some frequencies contaminate the spectrum of stator current, a proper band of frequency for covering fault frequency cannot be obtained. Therefore, more sensors and calculations need to be installed to allow an efficient fault detection process. In [11], an online unbalanced rotor fault detection based on a combination of time and frequency features of current and vibration signals were proposed. The authors considered Fourier and Hilbert transforms, and envelop detection (ED). The main limitations of this work are the need to access both current and vibration signals, and to perform several complicated feature extraction techniques. In [12], an enhanced field-oriented control approach was proposed for induction machines to detect the unbalanced rotor fault. The approach is capable of detecting the so-called high-resistance connections and consequently regulating the negative-sequence current.

In a recent research [13], an adaptive fault diagnosis technique for wind power gearbox was proposed. The researchers presented a Modified Laplacian of Gaussian filter (MloG).
Their method involves some pre-processing followed by a marginal envelope spectrum entropy technique to measure the fault characteristics. The drawback is that the pre-processing may lead to failed diagnosis of small faults. Other popular algorithms include envelope analysis based on Hilbert transform (ENV) [14], modulation signal bispectrum [15], and extend Park's vector approach [16]. A popular energy operator called Teager-Kaiser energy operator (TKEO), originally proposed in [17], has been widely used for machinery fault diagnosis, including gearbox [18] and bearing faults [19]. In [20], TKEO was used to demodulate the stator current and removing the main component of the current. However, it still may destruct adjacent components, associated to fault, which consequently decreases the detection accuracy. A frequency-based method proposed in [21] that requires calculating two spectrograms using fixed-length windows to segment the spectrograms. Since this method solely relies on FA, it may fail in low frequency resolutions.

Recently, the tendency toward using deep neural networks for induction machine fault diagnosis has been increased. A one-dimensional convolutional neural network (1D-CNN) was proposed in [22] to identify, quantify, and localise bearing damage. In [23], a deep learning network was proposed that learns the adaptive features without using prior knowledge. The network was trained to develop features that can distinguish different fault categories. A semi-supervised deep learning technique was proposed by Razavi-Far et al. for diagnosis of gear fault [24]. Their method is capable of diagnosing multiple defects including simultaneous ones in a gearbox directly connected to an induction machine shaft. A time domain current analysis approach was proposed in [25] for diagnosis of broken rotor bars in squirrel-cage induction machines. This method extracts histogram of oriented gradient (HoG) features from input signals which are then fed to a neural network for classification. Due to high computational complexity, this method is not suitable for real-time monitoring applications. In general, deep learning-based methods rely on a large-scale dataset to be trained. This normally requires a powerful computer due to a high computational complexity involved. Once the trained network is obtained, the model can be implemented for online condition monitoring and/or as a digital twin in real-time settings. However, if the working conditions of the machine change, then, the network should be re-trained according to the relevant training dataset.

A common challenge in the existing research is that when the supply and fault spectra of the current signature in IMs have significant frequency overlap, frequency-based methods fail to detect the fault. In order to tackle this issue, in this study we employ the singular spectrum analysis (SSA) technique. SSA is a powerful time-series analysis technique which is able to decompose a time-domain signal into several components with different trends. Despite the frequency overlap, SSA is able to calculate these trends in the eigenvalue spectra (rather than the frequency spectra) leading to finding a subspace where the fault component can be recognised. Interestingly, the obtained eigen-space also contains information about the frequency content of the data. For example, a periodic component in the data is reflected as a pair of equal eigenvalues. These features provide the flexibility to design and incorporate some data-driven filters along with existing prior knowledge about the input data to detect a specific trend, e.g., fault 
component, in the data. The aim of this study is to introduce an efficient SSA-based method both in terms of diagnosis' accuracy and computational complexity. We first use SSA to decompose the current signature into its encompassing components. Considering that the supply frequency of the current signature is known, we propose a sequence of simple grouping and pruning steps (known as data-driven filters) to identify the fault. This contribution is complemented by proposing a fast peak localisation algorithm which is then combined with the aforementioned SSA-based method to increase the fault diagnosis performance.

The rest of the paper is organised as follows. Next, the mathematical description of SSA method is given. The proposed approach in this study includes three key parts. First, an SSA-based method is proposed in Section III for classification of healthy and faulty signals. Second, a fault localisation technique along with the mathematical details is provided in Section IV. Third, the procedure to integrate these two methods in a recursive manner is described in Section V. In Section VI, the experimental set up and associated results are provided. Finally, the discussion and conclusion are drawn in Sections VIII and VII, respectively.

\section{Singular Spectrum Analysis}

\section{A. Background}

SSA is a model-free spectral estimation method, which decomposes a given signal into several interpretable components including slowly varying trends, oscillatory components, and unstructured noise. It is considered as a signal processing approach which works based on time series analysis and multivariate statistics principles. SSA is able to recognise different trends in the eigenvalue spectra irrespective of the frequency overlap among such components. This leads to finding a new subspace containing more information about the underlying components [26]. Although SSA has been widely used as a standard tool in climatic and meteorological time series analysis, nonlinear physics, and biomedical engineering, there is little work carried out to study its applicability for fault detection in IMs. In a study reported in [27], a vibrationbased method was used to build a baseline space from the feature space for fault detection in rolling element bearings. In contrast to [27], our proposed method here is data-driven, is applied to current signals and does not require any baseline space. In fact, our work is inspired by a series of recent SSAbased studies for separation and denoising biomedical signals such as Electromyography (EMG) and Electrocardiography (ECG) [28]-[32]. These works have proven that SSA is able to precisely detect and extract spectral components of interests event if they are closely located in the frequency domain and are partially overlapped.

\section{B. Mathematical description of SSA}

In essence, SSA decomposes the data into a number of components, classified as slow-varying, oscillatory, and unstructured, without leaving the current domain. This is done through two major stages, i.e., decomposition and reconstruction. Assume the input real-valued time series is represented by the vector $\mathbf{y}=\left[y_{1}, y_{2}, \ldots y_{n}\right]^{T}$ of size $1 \times n$. The corresponding trajectory matrix denoted by $\mathbf{Y}$ can be represented as follows:

$$
\mathbf{Y}=\left[\begin{array}{cccc}
y_{1} & y_{2} & \ldots & y_{m} \\
y_{2} & y_{3} & \ldots & y_{m+1} \\
\vdots & \vdots & & \vdots \\
y_{l} & y_{l+1} & \ldots & y_{n}
\end{array}\right]
$$

where $1<l<n$ is an integer, called windows length, and $m=n-l+1$. In fact, the trajectory matrix is obtained by constituting a map of sequential lagged vectors of length $l$. The next step is applying the singular value thresholding (SVD) to the covariance matrix $\mathbf{C}=\mathbf{Y} \mathbf{Y}^{T}$. Assume that $\lambda_{1}, \lambda_{2}, \lambda_{3}, \ldots, \lambda_{l}$ are singular values of $\mathbf{C}$ arranged in decreasing order so that $\lambda_{1} \geq \lambda_{2} \geq \ldots \geq \lambda_{l} \geq 0$. Further, $\mathbf{e}_{1}, \mathbf{e}_{2}, \ldots, \mathbf{e}_{l}$ are their associated eigenvectors. Every eigenvalue together with the associated eigenvector reconstructs one specific component of the original time series. Projecting the time series onto the direction of each eigenvector yields the corresponding temporal principal component (PC). If $\mathbf{v}_{i}=\mathbf{Y}^{T} \mathbf{e}_{i} / \sqrt{\lambda_{i}}$, then the SVD of the trajectory matrix can be written as

$$
\mathbf{Y}=\mathbf{Y}_{1}+\mathbf{Y}_{2}+\mathbf{Y}_{3}+\cdots+\mathbf{Y}_{d}
$$

where $d=\arg \max \left\{\lambda_{i}>0\right\}$ and $\mathbf{Y}_{i}=\sqrt{\lambda_{i}} \mathbf{e}_{i} \mathbf{v}_{i}^{T}$. The set $\left(\sqrt{\lambda_{i}}, \mathbf{e}_{i}, \mathbf{v}_{i}\right)$ is called $i$-th eigentriple. The eigentriples contain information about the frequency content of the data. For example, a periodic component in the data is reflected as a pair of (approximately) equal eigenvalues in the output of SSA. Moreover, the highest peaks in the Fourier transform of the corresponding eigenvectors are related to the frequency of the periodic component [30]. Generally, most of the important information about the signal is buried in the components corresponding to larger $\lambda_{i}$ 's. However, this is not always the case and smart grouping techniques (known as data-driven filters) must be adopted to extract the expected information from the signal.

\section{Proposed SSA-BASEd Fault Detection}

In general, the grouping procedure in SSA is to split the set of indices $i \in\{1,2, \cdots d\}$ into $k$ disjoint subsets $I_{1}, I_{2}, \cdots I_{k}$, where each subset consists of indices of $p$ eigenvalues $I=$ $\left\{i_{1}, i_{2}, \cdots i_{p}\right\}$. Each eigenvalue in the decomposition stage is equal to the variance of the signal in the direction of the corresponding PC. Larger eigenvalues correspond to signal components with greater energy. Therefore, one can divide the eigenvalues into two subspaces and conclude that the largest eigenvalues belong to the signal subspace, and interpret the smallest eigenvalues as noise. However, a greater number of subspaces can be obtained depending on different groups of eigenvalues, each interpreting a specific trend in the signal. It is worth noting that there are no general rules for grouping. However, researchers define different grouping strategies depending on the special requirements of the applications. In other words, a priori knowledge about the problem is usually used to design the right grouping technique.

Here, we assume that the input signal $\mathbf{y}$ is the stator current signature decomposed using SSA. Given the knowledge about supply component and the effects of faults, we propose to apply the following criteria to reject most unrelated components and to identify the fault:

- Coarse subspace rejection: In general, eigenvalues related to signal components are located in the lower subspace 
while those corresponding to unstructured noise belong to higher subspace. To preserve the lower subspace and reject the higher subspace, the following criterion is used. Any component corresponding to the eigenvalue $\lambda_{i}$ that satisfies $i>\tau$ via (4) is rejected:

$$
\tau=\arg \min _{a}\left\{\frac{\sum_{j=1}^{a} \lambda_{j}}{\sum_{j=1}^{l} \lambda_{j}}>\alpha\right\}
$$

where $\alpha$ determines a percentage of total variance in the signal, and $a$ is defined as the number of eigenvalues whose overall variances are above this percentage. A small $\alpha$ is not desired as it leads to rejection of a great number of eigenvalues which may include the fault component. Hence, it is rational to select a large $\alpha$ to avoid rejection of fault component and only remove unwanted higher subspace (equivalent to high frequency noise). Following the literature [30], [32], and based on our experimental verification, which will be represented in Section VI, we select $\alpha=0.95$ in this study.

- Periodic/Quasi-periodic component extraction: One interesting and useful property of SSA is that it produces "eigenvalue pairs" associated to any periodic or oscillatory time series component in the signal [30]. On the other hand, it is formerly known that both rotation of the rotor and the experienced fault lead to periodic/quasiperiodic components (appearing as eigenvalue pairs) in the decomposed signal. Thus, SSA seems to be appropriate for extracting these components. Figure 3 depicts an example plot of eigenvalues for both healthy and faulty time series, which gives an indication of the common/distinct components between the two. It is evident from this figure that the first four largest eigenvalues are pair-wise similar in both healthy and faulty signals. These eigenvalue pairs correspond to the 1st and the 3rd harmonics of the current signal, respectively, which are known to exist in both healthy and faulty conditions. The remaining eigenvalues are to be grouped appropriately to find those corresponding to the fault. In order to find suitable grouping criteria, the following characteristics should be considered: 1) noise can also generate equal eigenvalue pairs, 2) eigenvalue pairs associated to quasiperiodic components are not exactly equal, and 3) the corresponding eigenvectors have almost the same frequency amplitude. Assuming any pair of eigenvalues $\left(\lambda_{i}, \lambda_{j}\right)$, we propose the following conditions which must be met:

1) $(i, j)$ satisfies (4).

2) $\left|1-\frac{\lambda_{i}}{\lambda_{j}}\right|<\beta$.

3) $\left|1-\frac{\mathcal{F}_{P}\left(\mathbf{e}_{i}\right)}{\mathcal{F}_{P}\left(\mathbf{e}_{j}\right)}\right|<\gamma$, where $\mathcal{F}_{P}\left(\mathbf{e}_{i}\right)$ is the peak value of eigenvector $\mathbf{e}_{i}$ in the frequency domain.

The value of parameter $\beta$ determines the degree of periodicity of the signal component associated to $\left(\lambda_{i}, \lambda_{j}\right)$. $\beta=0$ corresponds to exact periodic components, while $\beta=1$ does not enforce any periodicity at all. In reality, both supply and fault components are periodic, thus, we empirically select $\beta=0.1$ to preserve any components with this characteristic. Likewise, $\gamma=0.4$ is selected to preserve eigenvector pairs corresponding to components whose frequency amplitudes are within a specific limit. In Section VI, we will provide an experiment to discuss how these values are empirically selected.

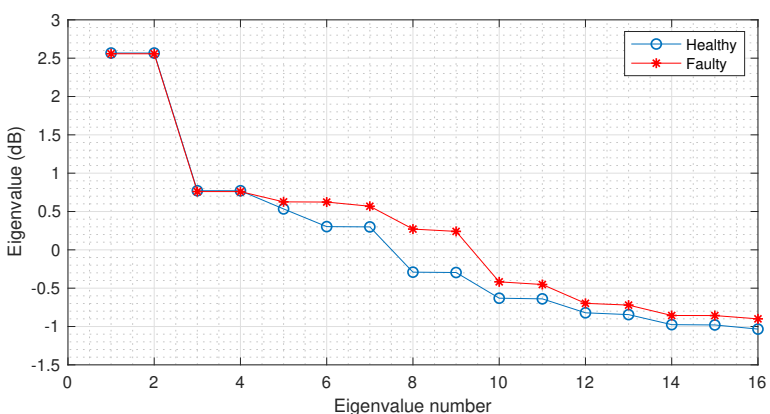

Fig. 3. Sorted eigenvalues in descending order. It is evident that the first two largest pairs of eigenvalues are exactly similar for Healthy and Faulty time series.

- Fine subspace rejection: In order to cope with the situations of faults with associated frequencies close to 50 $\mathrm{Hz}$ (high rotor speed), a fine subspace selection is further conducted. To do this, the frequency associated to the peak value of the Fourier spectrum in the remaining eigenvectors $\left(f_{i,(i=2, \ldots l)}\right)$ are compared to that for the eigenvector associated to the largest eigenvalue $\left(f_{1}\right)$. Then, the components with frequencies more than the largest eigenvalue frequency are omitted (i.e. $f_{i}>f_{1}$ ). Note that the Fourier spectrum is calculated from the eigenvectors and there is no need to reconstruct the principle components which is an expensive operation.

- Low-power component rejection: The remaining components so far are most likely to include the main rotatory and fault components plus a few low-energy noise-related ones. In order to reject all other components and only keep the supply and the fault, we propose the following criterion that calculates the relative energy of the remaining eigenvectors:

$$
\kappa=\left\{i \mid \frac{\mathcal{F}_{P}\left(\mathbf{e}_{i}\right)^{2}}{\left\|\mathcal{F}\left(\mathbf{e}_{i}\right)\right\|_{2}^{2}}>\eta\right\}
$$

where $\eta=0.65$ is a constant selected empirically with further details provided in Section IV. According to working principle of IMs, the main rotatory component centered at $50 \mathrm{~Hz}$ has the highest energy in the current signature's spectrum [1]. The fault component, though, has far less energy compared to the rotatory one but still has a higher energy compared to noise and other unwanted components. Therefore, the eigenvalues that fall into the subset $\kappa$ are preserved.

After applying all the above steps, if the number of remaining eigenvalues is more than ONE, the signal is classified as faulty otherwise it is regarded as a healthy signature. The pseudo-code of the proposed algorithm is given in Algorithm 1. Four constants in the grouping stage, i.e. $\alpha, \beta, \gamma, \eta$, are selected empirically and remained unchanged through the entire procedure.

\section{Proposed Fault Localisation Method}

The SSA-based method is only able to classify healthy and faulty signatures not to locate the fault. In this part of the paper, we propose a heuristic algorithm called fast peak localisation (FPL) that searches over the peaks of power spectrum of the extracted faulty component by SSA to locate the fault. Details of the proposed method is given as follows. 


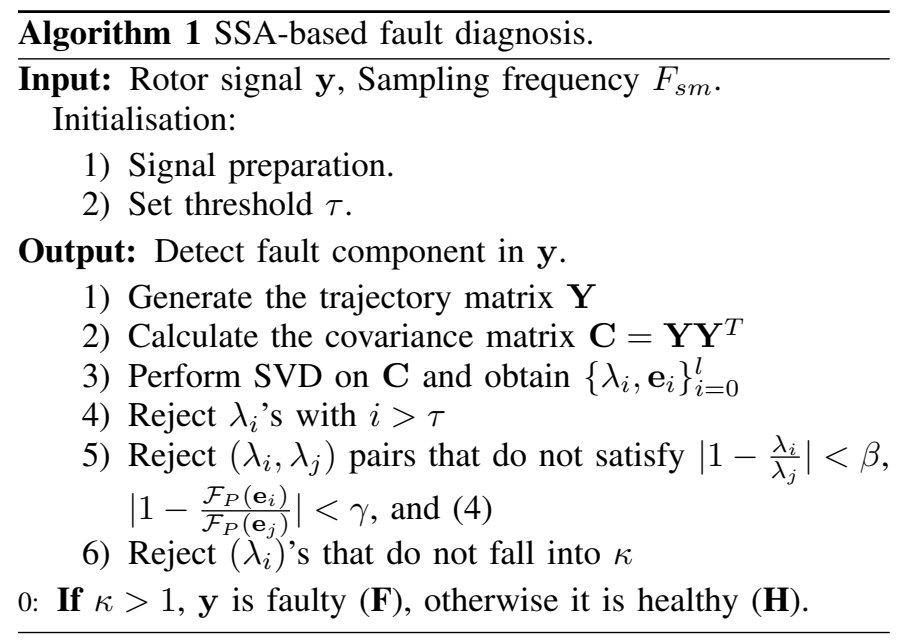

Recall from Section II-B, let us define the decomposed faulty component of current time series by $\hat{\mathbf{y}}=\left[\hat{y}_{1}, \hat{y}_{2}, \ldots \hat{y}_{n}\right]^{T}$ of size $1 \times n$. We denote the $n$-point non-negative frequency spectrum $\mathbf{x}=\left[x_{1}, x_{2}, \ldots, x_{k}, \ldots, x_{n}\right]^{T}$ in which every point can be calculated using FFT as follows [33]:

$$
x_{k}=\left|\sum_{m=0}^{n-1} \hat{y}_{m} e^{-j 2 \pi k m / n}\right| \quad k=0, \ldots, n-1
$$

where $e^{j 2 \pi / n}$ is a primitive $n$-th root of one. By definition, $x_{k}$ is a peak (local maximum) if it is not smaller than its adjacent samples, i.e., $x_{k}>x_{k-1}$ and $x_{k}>x_{k+1}$. Differentiation (derivative) is the traditional method to find the maximum and minimum values of a function [33]. Nevertheless, the presence of random noise in real-life signals normally yields many false zero-crossing if this technique is used. The typical solution is to smooth the input signal with some low-pass filters which usually destroys the original signal samples at the same time. This would be a major drawback in our work where the fault could be very small and signal smoothing might completely remove the fault peak. To avoid this, we omit the traditional mathematical approach and rather develop a computerised algorithm to check all the samples in the spectrum and find the local peaks. We simply define a local peak at the highest

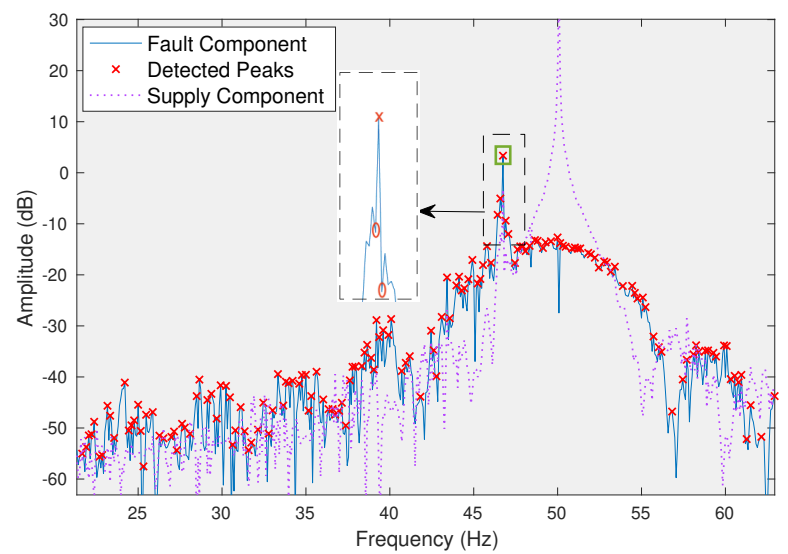

Fig. 4. Two spectral components of a current signal with $R_{u n b}=0.003$ p.u. and $1450 \mathrm{rpm}$. The supply (purple) and fault (blue) components are separated using the proposed SSA method. The red crosses show the detected peaks using the proposed FPL technique. The green rectangular indicates the location of fault at $f=46.74 \mathrm{~Hz}$. A sample peak location area is enlarged for better visibility where the valleys are identified by red circles.

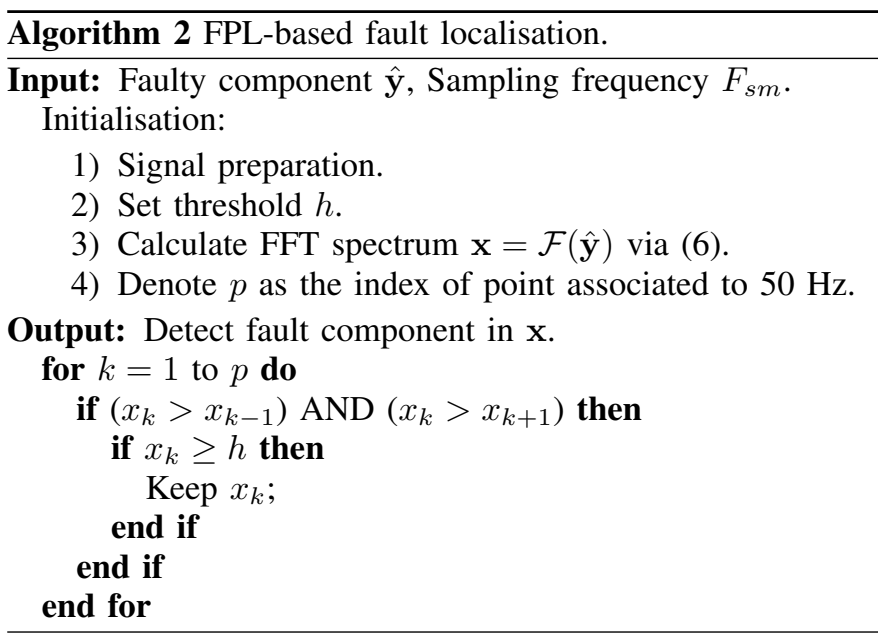

point between two valleys. Therefore, if the neighbouring samples around a point is identified as valleys then it should be marked as a peak. This is done by sweeping through all the samples and comparing the amplitudes of every sample with its neighbours. A local peak is flagged once value of a sample is not smaller than its adjacent samples. It is worth to mention that this process is applied to the fault component that was already extracted by SSA in the previous step, and not directly to the Fourier spectrum of the original current signature. In other words, the proposed SSA-based method identifies a subspace where fault can be detected properly. Then, this subspace is used by the FPL-based method to localise the fault. Figure 4 illustrates the results of peak localisation (depicted by red crosses) for a sample of faulty component (depicted in blue). For better visualisation and comparison, we have also included the supply component (depicted in purple) in this figure. It is found from Figure 4 that a faulty spike has appeared at $f=46.74 \mathrm{~Hz}$. Notably, Figure 4 reveals that the decomposed components using SSA allow localisation of fault using the proposed FPL method, while power spectrum of the input current signature does not give such indication (Figure 1). FPL algorithm should automatically identify and locate such faults. In order to do this, FPL compares all the extracted local peaks within [0 50] Hz, against a predefined threshold (denoted by $h$ in Algorithm 2). If any local peak falls above the threshold, it is flagged as fault and its location is recorded. This process is repeated for all the samples to detect any existing spike which may represent a fault. The full procedure of this method is presented in Algorithm 2.

\section{Proposed Integrated SSA-FPL Method}

The proposed methods, described in the previous sections, are capable of diagnosing current signature of IMs. While FPL is very simple, suitable for real-time fault localisation, SSAbased method is slower but able to decompose the current signature into finer frequency contents which might be hidden under the main frequency sideband. It is therefore rational to combine these two methods in a way to improve the diagnosing performance, particularly at a challenging case of higher rotor speed and low fault severity. In this part of the paper, we describe a topology to integrate SSA and FPL based on an adaptive strategy as depicted in Figure 5. In this approach, as observed from this figure, the current signature is captured 
first and then it is diagnosed with SSA method based on a predefined window size. If the outcome is detection of a faulty component, then, the corresponding component is forwarded to FPL for fault localisation. However, if SSA is unable to detect any fault, the signal might either be healthy or include a small fault. In order to increase the accuracy, we repeat the SSA diagnosis, this time with a larger window size (equivalent to higher eigenvalue spectrum resolution). This process is repeated for several iterations until the location of fault is found or the signal is classified as healthy. The advantage of this procedure is that as the window size increases, finer spectrum contents can be decomposed, therefore, detection accuracy could be increased. The negative impact, though, is slight increase in the processing time which is negligible as it is only fraction of a second.

To summarise the roles and effects of both SSA and FPL, we refer back to Figure 1 where a sample of faulty signature with $s=0.03$ and $R_{u n b}=0.003$ p.u. was given. According to this figure, FPL fails if applied directly to this signal. However, applying SSA to this signal will extract the faulty component from the original spectrum (Figure 4 - solid blue curve). If FPL is applied to the extracted component, i.e., to the output of SSA (Figure 5), the fault will be correctly localised (Figure 4 - green box). Such combination of the two methods can obviously improve the overall diagnosis performance which will be supported by our experiments in the next section.

\section{EXPERIMENTAL RESULTS}

In order to evaluate the performance of the proposed approaches, fault diagnosis of unbalanced rotor winding in WRIM by means of stator current signature is investigated. A three-phase $250 \mathrm{~W}, 50 \mathrm{~Hz}, 400 \mathrm{~V}$, four-pole, $1360 \mathrm{rpm}$ WRIM digitally controlled by brake system is tested in different operational slips and fault severity. The severity of fault is controlled by additive serial resistance $\left(R_{u n b}\right)$ in the rotor winding of WRIM as shown Figure 6. In this regard, extensive range of resistances and slips are considered to show the validity of the proposed approach in different conditions. The details of system setup, installed in our laboratory at Shahrood University of Technology, is illustrated in Figure 6 . The numerical analyses in this work were conducted in MATLAB R2019a environment, on a MacBook Pro laptop with Quad-Core Intel Core i5 $2.4 \mathrm{GHz}$ and $8 \mathrm{~GB}$ of Memory. Two sets of data were collected to validate the performance of the proposed approach. First dataset (D1) is composed of three different slips $(s)$ of WRIM 0.06 (1410 rpm), 0.047 $(1430 \mathrm{rpm})$, and $0.033(1450 \mathrm{rpm})$, each having 18 trial signals for faulty and 9 trial signals for healthy class in two

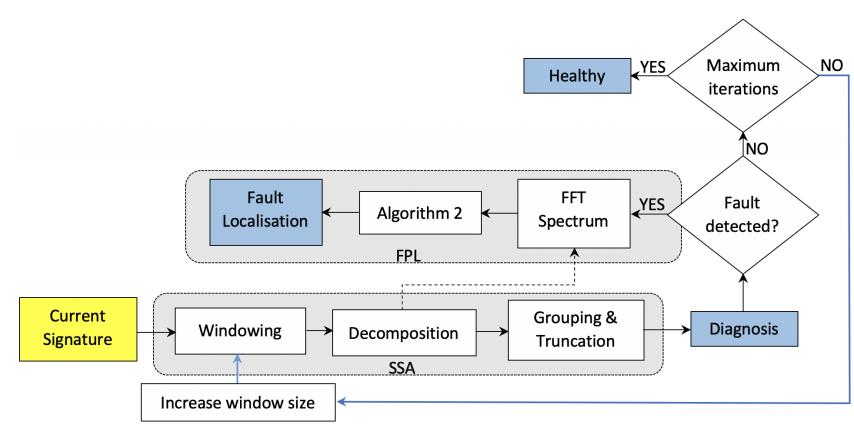

Fig. 5. Flowchart of the proposed integrated SSA-FPL approach.

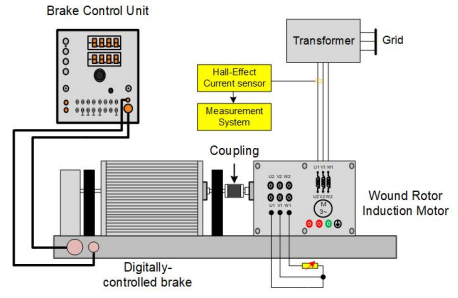

(a)

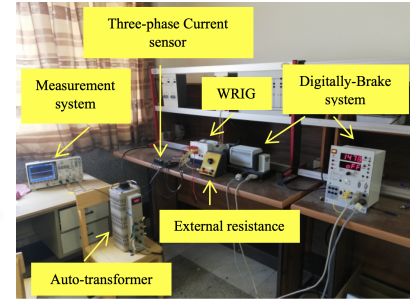

(b)
Fig. 6. Experimental test-rig. (a) schematic; (b) set up environment.

different fault cases $\left(R_{u n b}=0.156,0.468\right.$ p.u. $)$. This dataset includes 27 healthy signal and 54 faulty signals. In the second dataset (D2), the speeds were 1370, 1410, and $1450 \mathrm{rpm}$, as well as $1477 \mathrm{rpm}$ for the no-load (NL) case. Various faults at $R_{u n b}=0.003,0.031,0.093,0.156$, and 0.218 p.u. were considered. Totally, there are 900 faulty and 180 healthy signals of length 40000 samples in this dataset.

A set of well-established performance measures, i.e., Accuracy, Precision, Recall and Fl-score, have been considered to evaluate the performance of the proposed methods. These metrics are calculated as follows:

$$
\begin{aligned}
& \text { Accuracy }=\frac{\mathrm{TP}+\mathrm{TN}}{\mathrm{TP}+\mathrm{FP}+\mathrm{FN}+\mathrm{TN}} \\
& \text { Precision }=\frac{\mathrm{TP}}{\mathrm{TP}+\mathrm{FP}} \\
& \text { Recall }=\frac{\mathrm{TP}}{\mathrm{TP}+\mathrm{FN}} \\
& \mathrm{F} 1=\frac{2 \times \text { Recall } \times \text { Precision }}{\text { Recall }+ \text { Precision }}
\end{aligned}
$$

where TP, TN, FN, and FP, denote True Positive, True Negative, False Negative, and False Positive, respectively.

In the first experiment, we evaluated the performance of the SSA-based method and compared it with a simple Fourier analysis on D1 dataset. The diagnosis results using these methods are given in Table I. It is found from this table that SSA-based approach performs very well and have recognised all healthy signals and failed to detect only $2 \%$ of the faulty signals. Fourier analysis shows perfect detection of healthy signals, but weaker performance in detecting faulty signals. The $5 \%$ failure mainly corresponds to signals with small faults (smaller $R_{u n b}$ ) as expected. This experiment verifies the effectiveness of SSA-based method for the purpose of fault diagnosis from motor current signature. However, more challenging conditions are to be explored next.

We used D2 dataset which includes more samples of current signatures with wider range of faults including a very small fault $\left(R_{u n b}=0.003\right.$ p.u. $)$ as well as a high speed at 1450 rpm. Figure 7 explains how the amplitude of fault frequency

TABLE I

AVERAGE CLASSIFICATION ACCURACY WITH D1. $\boldsymbol{H}$ AND $\boldsymbol{F}$ REFER TO HEALTHY AND FAULTY, RESPECTIVELY.

\begin{tabular}{ccccc}
\hline \multirow{3}{*}{ Actual class } & \multicolumn{4}{c}{ Predicted class } \\
\cline { 2 - 5 } & \multicolumn{2}{c}{ SSA-based } & Fourier & Analysis \\
\cline { 2 - 5 } & $\boldsymbol{H}$ & $\boldsymbol{F}$ & $\boldsymbol{H}$ & $\boldsymbol{F}$ \\
\hline $\boldsymbol{H}$ & $100 \%$ & $0 \%$ & $100 \%$ & $0 \%$ \\
$\boldsymbol{F}$ & $2 \%$ & $98 \%$ & $5 \%$ & $95 \%$ \\
\hline
\end{tabular}




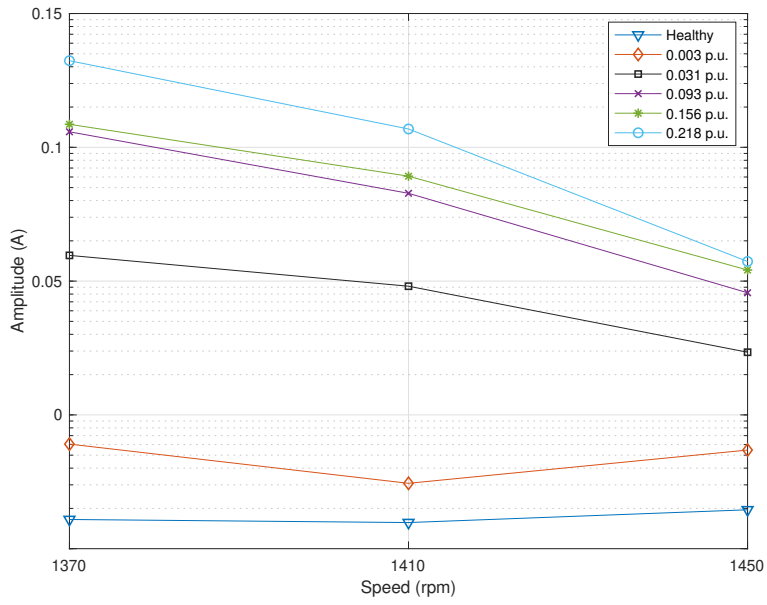

Fig. 7. Amplitude (power spectrum) of healthy and faulty current signatures versus various speeds. These values are obtained by calculating the fault frequency using (1) and by manually measuring the amplitude of the corresponding sample at both faulty and healthy signals spectrum.

component changes at various rotor speeds in this dataset. It is noticeable from this figure that the amplitude of fault with $R_{\text {unb }}=0.003$ p.u. is almost the same as that for healthy signatures. Therefore, it cannot be distinguished easily. Furthermore, based on observation from Figure 7, as the rotor speed increases the amplitude of fault component drops which makes the fault detection more challenging. Thanks to the spectral component analysis using SSA-based method most of these fault components can be recognised.

In Tables II and III, a detailed performance analysis of various faults and speeds can be found. These results were calculated with D2 dataset and provide insight on the portion of correctly diagnosed signals under various working conditions. According to these tables, both methods inevitably perform weaker at small faults and higher speeds while their performance is mostly around $100 \%$ in other conditions. As expected, the detection accuracies are considerably low at no-load (NL) condition. This is mainly due to minimum interaction between rotor and stator fields leading to significant modulation of fault and supply components. Nevertheless, SSA-FPL performs slightly better, especially when severity of fault increases. In general, when SSA is combined with FPL, the diagnosis performance is considerably increased. This is due to the fact that SSA-FPL repeats the spectrum analysis under various resolutions until diagnosing the fault which is followed by localisation using FPL.

In order to visualise how the accuracy changes at different rotor speeds and fault severity, we conducted an experiment with D2 dataset and recorded the detection accuracy accordingly. Figure 8 illustrates these results for SSA-FPL method. It is clearly observed from this figure that the proposed method performs very well for moderate cases, i.e. $R_{u n b}=0.031$, $0.093,0.156$, and 0.218 p.u.. However, the performance is lower with small fault $\left(R_{u n b}=0.003\right.$ p.u.). Except this case, SSA-FPL still achieves high accuracy under the same fault conditions but at lowest speed, i.e., $1370 \mathrm{rpm}$. It is generally observed that as the motor speed increases the accuracy falls moderately. According to Figure 8, the proposed method recorded nearly $100 \%$ accuracy of healthy signal detection in all speeds.

Generally, based on SSA theory, as the window size $l$
TABLE II

DIAGNOSIS RESULTS WITH D2 USING SSA-BASED METHOD UNDER DIFFERENT WORKING CONDITIONS.

\begin{tabular}{clccccc}
\hline$R_{\text {unb }}$ (p.u.) & Speed (rpm) & TP & TN & FP & FN & Accuracy $(\%)$ \\
\hline \multirow{5}{*}{0.003} & 1370 & 33 & 0 & 0 & 12 & 73 \\
& 1410 & 28 & 0 & 0 & 17 & 62 \\
& 1450 & 16 & 0 & 0 & 19 & 35 \\
& $1477(\mathrm{NL})$ & 5 & 0 & 0 & 40 & 11 \\
\hline \multirow{3}{*}{0.031} & 1370 & 45 & 0 & 0 & 0 & 100 \\
& 1410 & 43 & 0 & 0 & 2 & 95 \\
& 1450 & 40 & 0 & 0 & 5 & 84 \\
& $1477(\mathrm{NL})$ & 7 & 0 & 0 & 38 & 15 \\
\hline \multirow{3}{*}{0.093} & 1370 & 45 & 0 & 0 & 0 & 100 \\
& 1410 & 45 & 0 & 0 & 0 & 100 \\
& 1450 & 38 & 0 & 0 & 7 & 89 \\
& $1477(\mathrm{NL})$ & 3 & 0 & 0 & 7 & 16 \\
\hline \multirow{5}{*}{0.156} & 1370 & 45 & 0 & 0 & 0 & 100 \\
& 1410 & 45 & 0 & 0 & 0 & 100 \\
& 1450 & 42 & 0 & 0 & 3 & 93 \\
0.218 & $1477(\mathrm{NL})$ & 10 & 0 & 0 & 35 & 22 \\
\hline & 1370 & 44 & 0 & 0 & 1 & 97 \\
& 1410 & 45 & 0 & 0 & 0 & 100 \\
& 1450 & 45 & 0 & 0 & 0 & 100 \\
& $1477(\mathrm{NL})$ & 13 & 0 & 0 & 32 & 28 \\
\hline \multirow{6}{*}{0} & 1370 & 1 & 40 & 4 & 0 & 91 \\
& 1410 & 0 & 45 & 0 & 0 & 100 \\
& 1450 & 0 & 34 & 11 & 0 & 75 \\
& $1477(\mathrm{NL})$ & 0 & 29 & 16 & 0 & 64 \\
\hline
\end{tabular}

TABLE III

DIAGNOSIS RESULTS WITH D2 USING ITERATIVE SSA-FPL METHOD UNDER DIFFERENT WORKING CONDITIONS.

\begin{tabular}{|c|c|c|c|c|c|c|}
\hline$R_{\text {unb }}$ (p.u.) & Speed (rpm) & $\mathrm{TP}$ & TN & FP & FN & Accuracy (\%) \\
\hline \multirow{4}{*}{0.003} & 1370 & 38 & 0 & 0 & 7 & 84 \\
\hline & 1410 & 33 & 0 & 0 & 12 & 73 \\
\hline & 1450 & 31 & 0 & 0 & 14 & 69 \\
\hline & 1477 (NL) & 7 & 0 & 0 & 38 & 15 \\
\hline \multirow{4}{*}{0.031} & 1370 & 45 & 0 & 0 & 0 & 100 \\
\hline & 1410 & 45 & 0 & 0 & 0 & 100 \\
\hline & 1450 & 43 & 0 & 0 & 2 & 95 \\
\hline & 1477 (NL) & 10 & 1 & 0 & 35 & 22 \\
\hline \multirow{4}{*}{0.093} & 1370 & 45 & 0 & 0 & 0 & 100 \\
\hline & 1410 & 45 & 0 & 0 & 0 & 100 \\
\hline & 1450 & 45 & 0 & 0 & 0 & 100 \\
\hline & 1477 (NL) & 13 & 0 & 0 & 32 & 28 \\
\hline \multirow{4}{*}{0.156} & 1370 & 45 & 0 & 0 & 0 & 100 \\
\hline & 1410 & 45 & 0 & 0 & 0 & 100 \\
\hline & 1450 & 44 & 0 & 0 & 1 & 97 \\
\hline & 1477 (NL) & 14 & 0 & 0 & 31 & 31 \\
\hline \multirow{4}{*}{0.218} & 1370 & 45 & 0 & 0 & 0 & 100 \\
\hline & 1410 & 45 & 0 & 0 & 0 & 100 \\
\hline & 1450 & 45 & 0 & 0 & 0 & 100 \\
\hline & 1477 (NL) & 17 & 0 & 0 & 28 & 37 \\
\hline \multirow{4}{*}{0} & 1370 & 1 & 44 & 0 & 0 & 100 \\
\hline & 1410 & 0 & 45 & 0 & 0 & 100 \\
\hline & 1450 & 0 & 43 & 2 & 0 & 95 \\
\hline & 1477 (NL) & 0 & 39 & 6 & 0 & 86 \\
\hline
\end{tabular}

increases, finer spectral resolution can be achieved, leading to increased fault detection accuracy. In order to investigate the effects of the window size on diagnosis performance a typical experiment was conducted to demonstrate the tradeoff between the fault diagnosis and the computation time. We ran the SSA-based algorithm for various window sizes and recorded the computation time and the corresponding fault diagnosis accuracy. The result is shown in Figure 9. As noticed from this figure, when window size increases, both fault detection accuracy and computation times increase. However, the variations in the computation time is under one second even if very large window size is considered. This property has a positive implication when this system is 


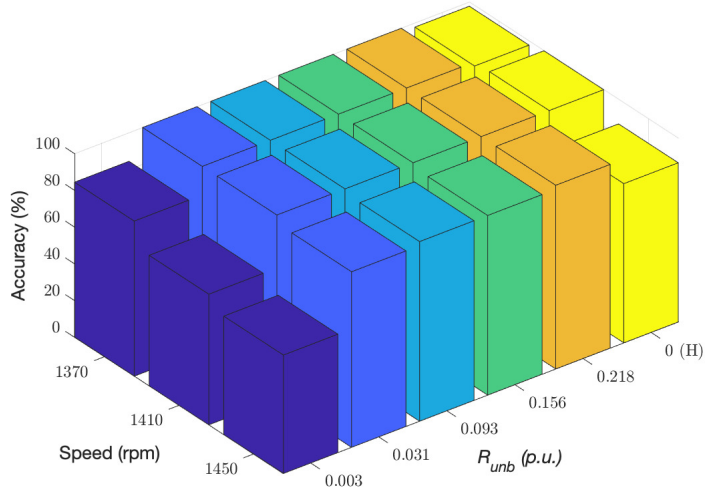

Fig. 8. Fault detection performance at various speeds and faults using SSAFPL method with D2 dataset.

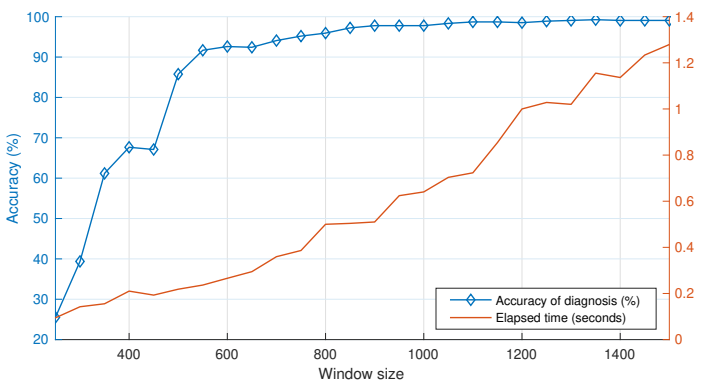

Fig. 9. Fault detection accuracy and elapsed time versus window size variation $l$. The left and right vertical axis show fault detection accuracy and computation time, respectively.

implemented in practice. It allows integrated SSA-FPL method to adaptively change the window size while the computation time might would not be significantly affected. For the sake of consistency, we have considered window sizes $l \approx 700$ as baseline in all our experiments.

Next, we conducted an experiment to verify the empirical selection of four key parameters (i.e., $\alpha, \beta, \gamma, \eta)$ in SSA grouping stage. During each sub-experiment, we fine-tune the value of one parameter in a specific range, e.g. $[0,1]$, while keeping other parameters fixed. Figure 10 demonstrates the recorded accuracy (\%) of fault detection by SSA, versus variations of these parameters. To ensure the generalisation of these parameters, we performed this experiment with D1 dataset, and then used the selected values through all experiments with both D1 and D2 datasets. According to Figure 10, a monotonic trend in accuracy is observed when these parameters vary. Moreover, the empirical selection of $\alpha=0.95, \beta=0.1$, $\gamma=0.4, \eta=0.65$ ensures a safe margin to provide highest diagnosis accuracy.

In order to observe and analyse the cases where SSAbased method fails, we depict in Figure 11 the accuracy of fault detection against various fault severities. This graph is for signals at a high speed, i.e., $1450 \mathrm{rpm}$, where the fault detection is more challenging. As seen from Figure 11(a) the poorest performance belongs to the cases with $R_{u n b}=0.003$ p.u. However, the accuracy is significantly improved when $R_{u n b} \geq 0.031$ p.u., and it monotonically continues until it reaches $100 \%$ for $R_{\text {unb }}=0.218$ p.u. Furthermore, Figures 11(b) and (c) show two sample signature spectrums where SSA succeeds and fails, respectively.
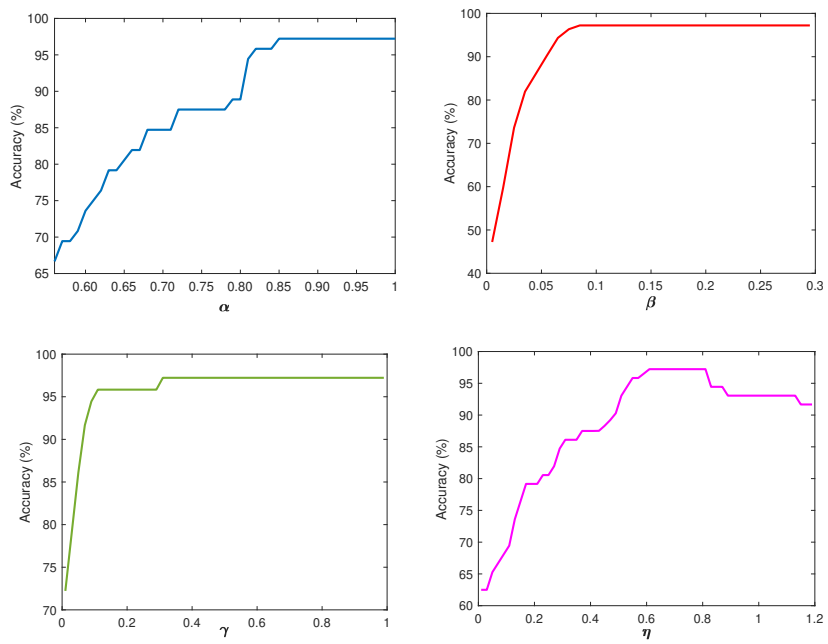

Fig. 10. Effects of changes in SSA parameters, i.e. $\alpha, \beta, \gamma, \eta$, on the diagnosis accuracy with D1 dataset.
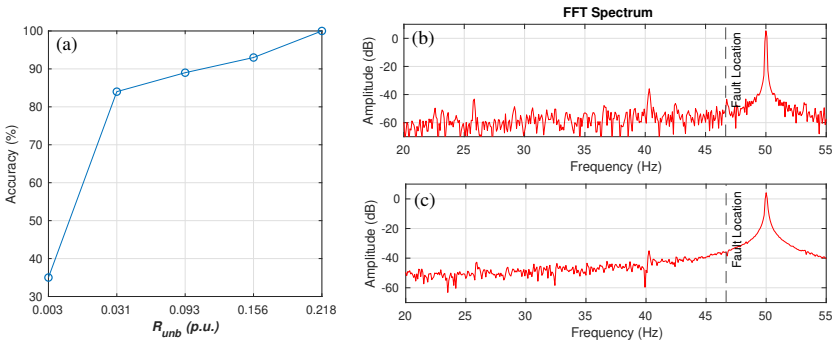

Fig. 11. SSA-based method's failure analysis: (a) fault detection accuracy at speed $1450 \mathrm{rpm}$ at different fault severities. Sample signatures of the same speed with $R_{u n b}=0.003$ where SSA-based method (a) succeeds and (b) fails.

In the proposed FPL algorithm, the parameter $h$ is empirically selected. Different choices of $h$ may influence differently on the diagnosis' performance. We conducted an experiment to study how changes in $h$ value can affect the fault localisation accuracy. This experiment was conducted on the entire signals in both D1 and D2 datasets where average accuracy against changes of $h \in\left[\begin{array}{ll}0 & 1\end{array}\right]$ was recorded. Figure 12 is a plot of the result of this experiment. As seen from this figure, highest accuracy is obtained for $h \approx 0.5$ indicating the best choice for $h$. It is worthwhile to mention that the trend of the graph in Figure 12 indicates the robustness of the proposed method against varying $h$.

In order to assess the quality of proposed fault localisation

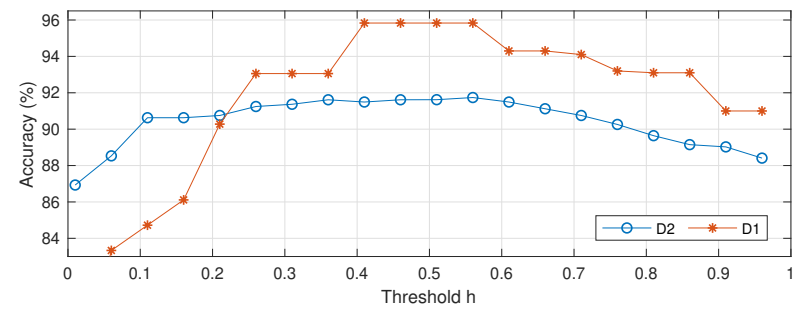

Fig. 12. Average accuracy of fault localisation against varying $h$, when FPL applied to both D1 and D2 dataset. 


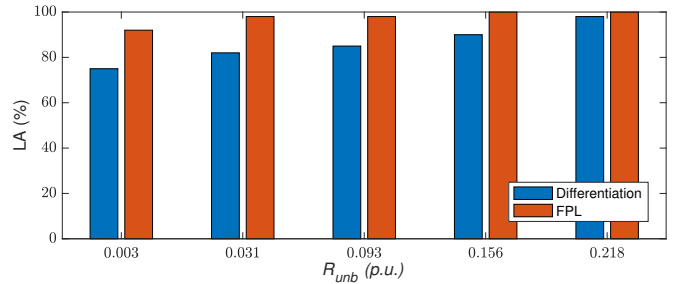

Fig. 13. Average localisation accuracy of different fault conditions after applying proposed FPL-based method and traditional differentiation. The results are averaged over all slips.

method (i.e. FPL) a performance measure is calculated and a comparison with traditional differentiation method is provided. Assuming that the number of faulty components and those of incorrectly localised for all rotor speeds are denoted by $N_{\text {total }}$ and $N_{\text {incorrect }}$, respectively. Then, the localisation accuracy (LA) is simply obtained via:

$$
\mathbf{L A}=\frac{N_{\text {total }}-N_{\text {incorrect }}}{N_{\text {total }}} \times 100 .
$$

The value of above metric is illustrated in Figure 13 for both methods. It is found from this figure that FPL-based method outperforms traditional differentiation method. This is particularly evident in fault conditions with small $R_{u n b}$ where differentiation technique does not perform well due to smoothing it applies to the signal.

Table IV compares the average performance metrics as well as running times when different methods applied to dataset D2. For comparison, we considered nine different methods, namely FA, DWT, (ENV [14], TKEO [20], KNN (k-nearest neighbour), MLP (multilayer perceptron), SVM (support vector machine), 1D-CNN [22], and LiftingNet [23]. As already mentioned, the proposed method (as well as FA, DWT, ENV and TKEO) is a data-driven technique which does not require a training phase as opposed to neural networks or traditional classifiers. It is designed based on the knowledge about the properties of the current signature such as supply frequency (which is known and fixed) and the frequency range in which the fault can potentially occur. Here, we compare various techniques with different working principles: classic machine learning (SVM, KNN and MLP), deep neural networks (1D$\mathrm{CNN}$ and LiftingNet), and data-driven time/frequency domains (FA, DWT, ENV, TKEO, SSA, and SSA-FPL). A 10-fold cross validation was applied for methods that require a training phase. As found from this table, SSA-FPL outperforms where it achieves highest accuracy, recall and F1. TKEO and LiftingNet achieve third and fourth highest accuracies, respectively, comparable to SSA-based method. The main reason that deep learning models do not perform very well is their reliance on large-scale training databases which are not always available. It is also observed that DWT performs slightly better than FA.

Table IV further shows the average computation time to diagnose the fault of a current signature under the same conditions for all methods. Both testing and training times are reported only for those methods that require such procedure. According to the table, FA is the fastest technique among data-driven methods where it can detect the fault within 0.15 seconds. Deep learning methods, i.e. 1D-CNN and LiftingNet require significant training time as expected.
TABLE IV

COMPARISON OF CLASSIFICATION RESULTS WITH D2 DATABASE USING DIFFERENT METHODS. COMPUTATION TIME IS PROVIDED FOR BOTH TRAINING (IF APPLICABLE) AND TESTING PHASES: TESTING/TRAINING.

\begin{tabular}{lccccc}
\hline Method & Accuracy & Precision & Recall & F1 & Time (s) \\
\hline FA & $85.80 \%$ & $97.52 \%$ & $82.12 \%$ & $89.16 \%$ & $0.15 /-$ \\
DWT & $87.10 \%$ & $96.42 \%$ & $85.83 \%$ & $90.82 \%$ & $0.33 /-$ \\
TKEO & $90.85 \%$ & $95.93 \%$ & $88.04 \%$ & $91.81 \%$ & $0.25 /-$ \\
ENV & $87.32 \%$ & $93.72 \%$ & $86.26 \%$ & $89.83 \%$ & $0.54 /-$ \\
KNN & $68.34 \%$ & $80.21 \%$ & $69.62 \%$ & $74.54 \%$ & $0.17 / 10.7$ \\
SVM & $75.24 \%$ & $83.86 \%$ & $71.04 \%$ & $76.91 \%$ & $0.09 / 3.41$ \\
MLP & $78.16 \%$ & $84.77 \%$ & $79.36 \%$ & $81.97 \%$ & $1.26 / 87.3$ \\
1D-CNN & $82.22 \%$ & $88.05 \%$ & $81.15 \%$ & $84.45 \%$ & $2.69 / 328$ \\
LiftingNet & $89.63 \%$ & $96.34 \%$ & $84.70 \%$ & $90.14 \%$ & $4.23 / 510$ \\
SSA-based & $91.37 \%$ & $98.25 \%$ & $91.42 \%$ & $94.71 \%$ & $0.22 /-$ \\
SSA-FPL & $94.57 \%$ & $99.07 \%$ & $94.53 \%$ & $96.75 \%$ & $0.28 /-$ \\
\hline
\end{tabular}

For further comparison and verification of the results, we calculated the relative differentiation (RD) of the detection results are calculated using:

$$
\mathbf{R D}=\frac{A_{F}-A_{H}}{\max \left\{A_{F}, A_{H}\right\}}
$$

where $A_{F}$ and $A_{H}$ represent the amplitudes of fault component and the associated frequency component in the healthy spectrum, respectively. We calculated average value of RD with D2 database at three differed rotor speeds. Figure 14 shows the results of this comparison where RD rates are depicted versus varying fault severity at i.e. 1370, 1410, and $1450 \mathrm{rpm}$ rotor speeds. This experiment can be performed only with methods with the ability to locate the fault. Therefore, we considered FA, ENV [14], and TKEO [20] to compare RD values. According to this figure, SSA-FPL demonstrates highest RD values among all methods. This is noticeable at fault with $R_{u n b}=0.031$ which is considered a challenging case. For severe faults, e.g. at $R_{u n b}=0.218$, the performance of TKEO is slightly less than SSA-FPL but comparable. Moreover, analysing all the RD values reveals that as the rotor speed increases the RD rate drops where the lowest rate occurs at fault with $R_{u n b}=0.003$ with $1450 \mathrm{rpm}$. The negative $\mathrm{RD}$ values obtained at this case is an indication of failing to correctly detect the fault due to its ultra-small amplitude and closeness to the main supply frequency. Notably, FA fails to diagnose small faults, particularly at high rotor speeds, while it performs promisingly in severe faults. We conclude that higher $\mathrm{RD}$ values, obtained using the proposed method, is mainly due to the fact that the proposed method does not apply any suppression on the frequency harmonics as opposed to other methods in this comparison.

\section{DISCUSSION}

Induction machine faults have many types, and the proposed method mainly focuses on the unbalanced rotor fault. Generally, low torque oscillations (LTOs), which occur because of defect in the drive-train of system, may lead to false alarm due to interaction with rotor asymmetry faults (RAFs). RAFs $\left(f_{R A F s}\right)$ and LTOs $\left(f_{L T O S}\right)$ stator current sidebands due to rotor asymmetry and rotational frequency of rotor can be observed as:

$$
\begin{aligned}
& f_{R A F s}=(1 \pm 2 k s) f_{s}, k=1,2, \ldots \\
& f_{\text {LTOs }}=f_{s} \pm k f_{r}=f_{s} \pm \vartheta(1-s) f_{s} / p, \vartheta=1,2,3,
\end{aligned}
$$



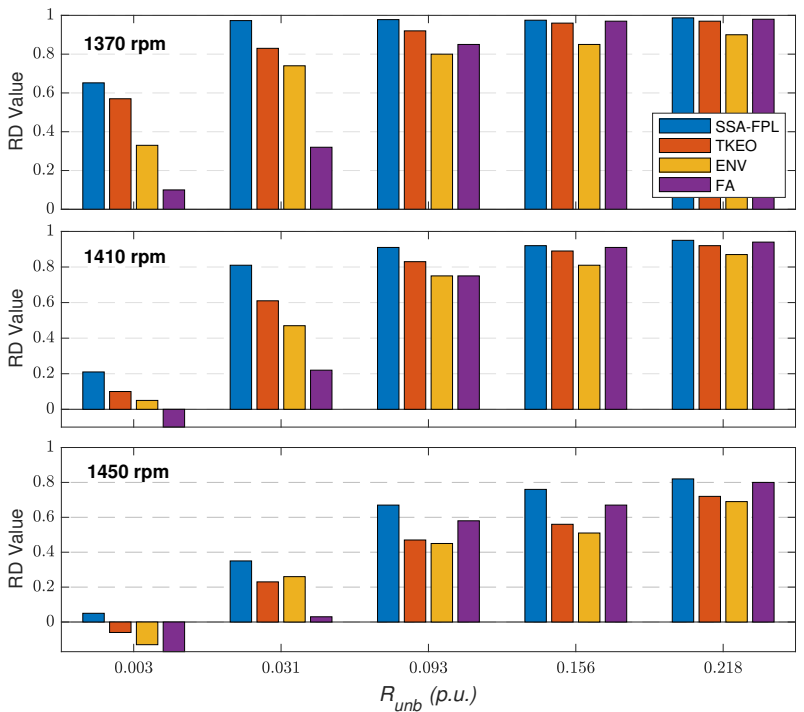

Fig. 14. Comparison of RD value calculated for the faults detected at various severity using three methods. Top, middle and bottom figures correspond to 1370,1410 and $1450 \mathrm{rpm}$ speeds, respectively.

where $f_{s}$ is supply frequency, $s$ is the slip and $p$ is the number of pole pair. The $f_{r}$ component is generated in the stator current of induction machines due to mechanical non-idealities in the motor, coupling or alignment issues. In this paper, the validity of proposed technique was tested with line-fed induction machines in different fault severities and load levels. In a line-fed induction machine, $f_{r}$ component would approach the RAFs characteristic frequencies often due to gearbox or other reduction coupling systems $\left(f_{\text {LTOS }}\right)$ which was not present in the drive-train of our system [34]. Therefore, it can be easily detected near the RAFs characteristic frequency. It has been reported that the distinction between LTOs and RAFs characteristic frequency in line-fed or inverter-fed induction machines in case of $2 f_{s} \approx f_{r}$ under the process characteristic or in the presences of reduction components (e.g. gearbox) can be carried out through other indices placed at the space harmonics or by start-up current and positive sequences of stator current [34], [35]. Therefore, LTOs can be feasibly tracked and detected in the spectrum through other indices. It is necessary to note that the proposed method cannot separate the effects of LTOs and RAFs under the load torque oscillations related to the process characteristic without considering other indices introduced in [34].

\section{CONCLUSION}

In this paper, a novel technique for unbalanced rotor fault diagnosis in induction machines was presented. The proposed method uses singular spectrum analysis to decompose the input current signal into several spectral components. A set of data-driven filters was proposed and utilised to detect the faulty component. In order to locate the fault, a fast peak localisation technique was applied to the extracted components by SSA. The two proposed methods were then combined and performed in a recursive mode. In order to quantify the extension of the rotor asymmetry, five different fault severities as well as a healthy condition were examined. The proposed method was tested under these conditions and the results were reported. Our extensive experiments has shown effectiveness of this method for unbalanced rotor fault diagnosis. The results suggest that proposed method is able to diagnose the input current signature within a quarter of a second on a general computer. Moreover, it is a data-driven and unsupervised approach, not requiring a training phase. Hence, it can be simply implemented in industrial applications where the MCSA is of concern. For future work, we aim to examine the performance of the proposed method to detect other fault types, such as broken rotor bar and bearing faults. Extending the proposed method to diagnose multiple faults as well as the ability to deliver the severity of the faults are of our future plans too.

\section{REFERENCES}

[1] A. Choudhary, D. Goyal, S. L. Shimi, and A. Akula, "Condition monitoring and fault diagnosis of induction motors: A review," Archives of Computational Methods in Engineering, vol. 26, no. 4, pp. 1221$1238,2019$.

[2] H. Henao, G. Capolino, M. Fernandez-Cabanas, F. Filippetti, C. Bruzzese, E. Strangas, R. Pusca, J. Estima, M. Riera-Guasp, and S. Hedayati-Kia, "Trends in fault diagnosis for electrical machines: A review of diagnostic techniques," IEEE Industrial Electronics Magazine. vol. 8, no. 2, pp. 31-42, 2014.

[3] Z. Zhao, F. Fan, W. Wang, Y. Liu, and K. Y. See, "Detection of stator interturn short-circuit faults in inverter-fed induction motors by online common-mode impedance monitoring," IEEE Transactions on Instrumentation and Measurement, vol. 70, pp. 1-10, 2021.

[4] M. H. Marzebali, S. H. Kia, H. Henao, G. Capolino, and J. Faiz, "Planetary gearbox torsional vibration effects on wound-rotor induction generator electrical signatures," IEEE Transactions on Industry Applications, vol. 52, no. 6, pp. 4770-4780, Nov 2016.

[5] A. Stefani, A. Yazidi, C. Rossi, F. Filippetti, D. Casadei, and G. Capolino, "Doubly fed induction machines diagnosis based on signature analysis of rotor modulating signals," IEEE Transactions on Industry Applications, vol. 44, no. 6, pp. 1711-1721, Nov 2008.

[6] Y. Gritli, A. Stefani, C. Rossi, F. Filippetti, and A. Chatti, "Experimental validation of doubly fed induction machine electrical faults diagnosis under time-varying conditions," Electric Power Systems Research, vol. 81, no. 3, pp. 751-766, 2011

[7] S. H. Kia, H. Henao, and G.-A. Capolino, "Fault index statistical study for gear fault detection using stator current space vector analysis," IEEE Transactions on Industry Applications, vol. 52, no. 6, pp. 4781-4788, 2016.

[8] S. H. Kia, "Monitoring of wound rotor induction machines by means of discrete wavelet transform," Electric Power Components and Systems, vol. 46, no. 19-20, pp. 2021-2035, 2018.

[9] D. Shah, S. Nandi, and P. Neti, "Stator-interturn-fault detection of doubly fed induction generators using rotor-current and search-coilvoltage signature analysis," IEEE Transactions on Industry Applications, vol. 45, no. 5, pp. 1831-1842, Sep. 2009.

[10] M. M. Rahman' and M. N. Uddin, "Online unbalanced rotor fault detection of an im drive based on both time and frequency domain analyses," IEEE Transactions on Industry Applications, vol. 53, no. 4, pp. 4087-4096, 2017.

[11] M. M. Rahman and M. N. Uddin, "Online unbalanced rotor fault detection of an im drive based on both time and frequency domain analyses," in 2015 IEEE Industry Applications Society Annual Meeting, 2015, pp. $1-8$.

[12] M. Mengoni, L. Zarri, Y. Gritli, A. Tani, F. Filippetti, and S. B. Lee, "Online detection of high-resistance connections with negative-sequence regulators in three-phase induction motor drives," IEEE Transactions on Industry Applications, vol. 51, no. 2, pp. 1579-1586, 2015.

[13] N. Duan, J. Wang, T. Zhao, W. Du, X. Guo, and J. Wang, "A novel adaptive fault diagnosis method for wind power gearbox," IEEE Access, vol. 9, pp. $11226-11240,2021$.

[14] M. A. el Malek, A. K. Abdelsalam, and O. E. Hassan, "Induction motor broken rotor bar fault location detection through envelope analysis of start-up current using hilbert transform," Mechanical Systems and Signal Processing, vol. 93, pp. 332-350, 2017.

[15] F. Gu, T. Wang, A. Alwodai, X. Tian, Y. Shao, and A. Ball, "A new method of accurate broken rotor bar diagnosis based on modulation signal bispectrum analysis of motor current signals," Mechanical Systems and Signal Processing, vol. 50-51, pp. 400-413, 2015.

[16] K. N. Gyftakis, A. J. Marques Cardoso, and J. A. Antonino-Daviu, "Introducing the filtered park's and filtered extended park's vector approach to detect broken rotor bars in induction motors independently from the rotor slots number," Mechanical Systems and Signal Processing, vol. 93, pp. 30-50, 2017. 
[17] H. Teager, "Some observations on oral air flow during phonation," IEEE Transactions on Acoustics, Speech, and Signal Processing, vol. 28, no. 5 , pp. 599-601, 1980.

[18] I. Antoniadou, G. Manson, W. Staszewski, T. Barszcz, and K. Worden, "A time-frequency analysis approach for condition monitoring of a wind turbine gearbox under varying load conditions," Mechanical Systems and Signal Processing, vol. 64-65, pp. 188-216, 2015.

[19] Y. Imaouchen, M. Kedadouche, R. Alkama, and M. Thomas, "A frequency-weighted energy operator and complementary ensemble empirical mode decomposition for bearing fault detection," Mechanical Systems and Signal Processing, vol. 82, pp. 103-116, 2017.

[20] M. Pineda-Sanchez, R. Puche-Panadero, M. Riera-Guasp, J. PerezCruz, J. Roger-Folch, J. Pons-Llinares, V. Climente-Alarcon, and J. A Antonino-Daviu, "Application of the teager-kaiser energy operator to the fault diagnosis of induction motors," IEEE Transactions on Energy Conversion, vol. 28, no. 4, pp. 1036-1044, 2013.

[21] M. Lopez-Ramirez, L. M. Ledesma-Carrillo, F. M. Garcia-Guevara J. Munoz-Minjares, E. Cabal-Yepez, and F. J. Villalobos-Pina, "Automatic early broken-rotor-bar detection and classification using otsu segmentation," IEEE Access, vol. 8, pp. 112 624-112 632, 2020.

[22] O. Abdeljaber, S. Sassi, O. Avci, S. Kiranyaz, A. A. Ibrahim, and M. Gabbouj, "Fault detection and severity identification of ball bearings by online condition monitoring," IEEE Transactions on Industrial Electronics, vol. 66, no. 10, pp. 8136-8147, Oct 2019.

[23] J. Pan, Y. Zi, J. Chen, Z. Zhou, and B. Wang, "Liftingnet: A novel deep learning network with layerwise feature learning from noisy mechanical data for fault classification," IEEE Transactions on Industrial Electronics, vol. 65, no. 6, pp. 4973-4982, June 2018.

[24] R. Razavi-Far, E. Hallaji, M. Farajzadeh-Zanjani, M. Saif, S. H. Kia, H. Henao, and G. Capolino, "Information fusion and semi-supervised deep learning scheme for diagnosing gear faults in induction machine systems," IEEE Transactions on Industrial Electronics, vol. 66, no. 8 , pp. 6331-6342, Aug 2019.

[25] C. G. Dias, L. C. da Silva, and W. A. Luz Alves, "A histogram of oriented gradients approach for detecting broken bars in squirrelcage induction motors," IEEE Transactions on Instrumentation and Measurement, vol. 69, no. 9, pp. 6968-6981, 2020.

[26] N. Golyandina, V. Nekrutkin, and A. A. Zhigljavsky, "Analysis of time series structure - ssa and related techniques," in Monographs on statistics and applied probability, 2001.

[27] H. Al-Bugharbee and I. Trendafilova, "A new methodology for fault detection in rolling element bearings using singular spectrum analysis," International Journal of Condition Monitoring, vol. 7, no. 2, pp. 26-35, 52017.

[28] S. Ferdowsi and V. Abolghasemi, "Semiblind spectral factorization approach for magnetic resonance spectroscopy quantification," IEEE Transactions on Biomedical Engineering, vol. 65, no. 8, pp. 1717-1724, Aug 2018.

[29] S. Sanei, T. K. M. Lee, and V. Abolghasemi, "A new adaptive line enhancer based on singular spectrum analysis," IEEE Transactions on Biomedical Engineering, vol. 59, no. 2, pp. 428-434, Feb 2012.

[30] F. Ghaderi, H. R. Mohseni, and S. Sanei, "Localizing heart sounds in respiratory signals using singular spectrum analysis," IEEE Transactions on Biomedical Engineering, vol. 58, no. 12, pp. 3360-3367, Dec 2011.

[31] M. Mortezaee, Z. Mortezaie, and V. Abolghasemi, "An improved ssabased technique for emg removal from ecg," IRBM, vol. 40, no. 1, pp. $62-68,2019$.

[32] S. M. Mohammadi, S. Kouchaki, M. Ghavami, and S. Sanei, "Improving time-frequency domain sleep eeg classification via singular spectrum analysis," Journal of Neuroscience Methods, vol. 273, pp. 96 - 106, 2016.

[33] A. V. Oppenheim and R. W. Schafer, Discrete-Time Signal Processing, 3rd ed. USA: Prentice Hall Press, 2009.

[34] H. Kim, S. B. Lee, S. B. Park, S. H. Kia, and G.-A. Capolino, "Reliable detection of rotor faults under the influence of low frequency load torque oscillations for applications with speed reduction couplings," in 2015 IEEE 10th International Symposium on Diagnostics for Electrical Machines, Power Electronics and Drives (SDEMPED), 2015, pp. 56-63.

[35] O. Guellout, A. Rezig, S. Touati, and A. Djerdir, "Elimination of broken rotor bars false indications in induction machines," Mathematics and Computers in Simulation, vol. 167, pp. 250-266, 2020, iNTERNATIONAL CONFERENCE on Emerging and Renewable Energy: Generation and Automation, held in Belfort, France on 4-6 July, 2017.

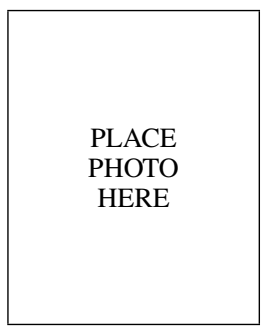

Vahid Abolghasemi (M'12-SM'17) is currently an Assistant Professor at the School of Computer Science and Electronic Engineering, University of Essex, UK. He received the Ph.D. degree from University of Surrey, Guildford, UK in 2011. During 2011-2019, he held academic positions at Brunel University and Shahrood Univeristy of technology. His main research interests include signal and image processing, compressive sensing, and dictionary learning. Most of his research findings and proposed solutions were published in high quality journals. He serves as a reviewer of various journals such as IEEE Transactions on Image Processing, IEEE Transactions on Signal Processing, IEEE Transactions on Neural System and Rehabilitation Engineering, etc.

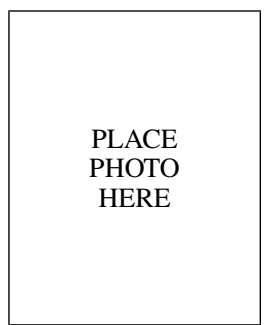

Mohammad Hoseintabar Marzebali received the B.Sc. degree in electrical power engineering from Mazandaran University, Mazandaran, Iran, in 2007, and the M.Sc. degree in electrical power engineering from Shiraz University of Technology, Shiraz, Iran, in 2010. In 2014, he received a scholarship from Campus France to be a joint Ph.D. as a Cotutelle Student between the University of Tehran, Tehran, Iran, and the University of Picardie "Jules Verne," Amiens, France. Currently, he is an Assitant Professor at Shahrood University of Technology. His research interests include fault diagnose in electrical machine and modular multilevel converters.

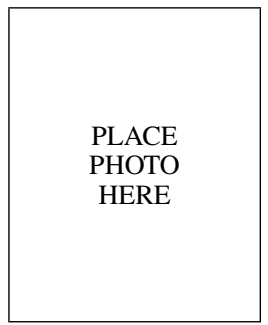

Saideh Ferdowsi (M'12) received her $\mathrm{PhD}$ from the University of Surrey in 2012. She is currently a Senior Research Officer at University of Essex, UK. Her main research interests are biomedical signal and image processing, blind source separation, spectrum analysis, and deep learning. She has been an active reviewer for Scientific Reports, IEEE Transactions on Biomedical Engineering, IEEE Transactions on Signal Processing, IEEE Transactions on Neural System and Rehabilitation Engineering journals. 